\title{
Upregulation of CPNE7 in mesenchymal stromal cells promotes oral squamous cell carcinoma metastasis through the NF-KB pathway
}

\author{
Xiaoli Ji (iD) ${ }^{1,2,3,9 凶}$, Tianyong Sun ${ }^{3}$, Shang Xie ${ }^{4}$, Hua Qian ${ }^{5}$, lixiang Song ${ }^{6}$, lihua Wang ${ }^{3}$, Hongwei Liu (D) ${ }^{7,9 凶}$ and Qiang Feng (D) ${ }^{3,8,9 凶}$
}

(c) The Author(s) 2021

A remarkable shift in Mesenchymal stromal cells (MSCs) plays an important role in cancer metastasis, but the molecular mechanism is still unclear. CPNE7, a calcium-dependent phospholipid-binding protein, mediates signal transduction and metastasis in many tumours. Here, we demonstrated that MSCs derived from OSCC (OSCC-MSCs) promoted the metastasis of OSCC cells by transwell assay and animal models through epithelial to mesenchymal transition (EMT) $(p<0.05)$. RNA-sequencing, ELISA, neutralizing antibody and CXCR2 inhibitor assay confirmed that CXCL8 secreted by OSCC-MSCs was associated with the upregulated expression of CPNE7 by immunohistochemical and western blotting $(p<0.05)$. This is mechanistically linked to the activation of CPNE7 to NF-KB pathway-induced metastasis, including phosphorylated p65 and IKBa. CPNE7 silencing inhibited metastatic abilities and the expression of CXCL8, phosphorylated p65, ІкBa, and p65 nuclear translocation by western blotting and immunofluorescence, while CPNE7 overexpression markedly promoted these events $(p<0.05)$. We also identified that Nucleolin could be bind CPNE7 and IKBa by co-immunoprecipitation. Together, our results suggest that upregulation of CPNE7 in MSCs interacted with surface receptor -Nucleolin and then combined with IKBa to promoted phosphorylated IKBa and $\mathrm{p} 65$ nuclear translocation to active NF-KB pathway, and then regulates CXCL8 secretion to promote the metastasis of OSCC cells. Therefore, CPNE7 in MSCs could be promising therapeutic targets in OSCC.

Cell Death Discovery (2021)7:294; https://doi.org/10.1038/s41420-021-00684-w

\section{INTRODUCTION}

Oral squamous cell carcinoma (OSCC) accounts for more than $90 \%$ of oral cancer and is one of the most prevalent cancers worldwide [1-3]. Despite advancements in treatment, metastasis is still the main cause of OSCC-related death [2]. In this process, tumour cells have to acquire an epithelial-mesenchymal transformation (EMT) phenotype, by which epithelial cells gain mesenchymal properties [4]. The earliest stage of EMT does not take place in metastatic progression but in pre-neoplastic lesions [5, 6]. Oral leukoplakia (OLK), with an incidence of $4.1 \%$ worldwide, is the most common pre-neoplastic lesion of OSCC $[7,8]$. Currently, the challenge is to prevent the metastasis of OSCC in the pre-neoplastic lesion, but the related mechanism remains unclear.

Mesenchymal stromal cells (MSCs) are critical components of the tumour microenvironment (TME) and play a crucial role in the metastasis of tumours [9-11]. MSCs, as progenitors of stromal cells, have been isolated from different stages of normal tissues, pre-neoplastic lesions, and cancer tissues [12-15]. In normal tissue, MSCs are in a quiescent state to maintain tissue architecture [16]. With the initiation of tumorigenesis, MSCs gradually begin to transition into a determined reprogrammed state accompanied by aberrant mutations in tumour cells $[9,10]$. The TME provides a niche for MSCs and tumour cells to modify or communicate with each other, in which confers a pro-migratory state of the TME $[14,15]$. Therefore, an increasing number of studies have focused on the question of whether pre-neoplastic or tumour-associated MSCs have distinct characteristics with a unique gene expression profile in a paracrine manner [17-19]. Studies have shown that MSCs derived from cervical intraepithelial neoplasia (precancerous lesion of the uterine cervix) can secrete several cytokines to sustain an anti-tumour microenvironment and block the progression of tumours [19], breast

\footnotetext{
${ }^{1}$ Department of Stomatology, Jinan Central Hospital, Cheeloo College of Medicine, Shandong University, No.105 Jiefang Road, Jinan 250013 Shandong, China. ${ }^{2}$ Department of Oral Mucosal Diseases, School and Hospital of Stomatology, Cheeloo College of Medicine, Shandong University \& Shandong Key Laboratory of Oral Tissue Regeneration \& Shandong Engineering Laboratory for Dental Materials and Oral Tissue Regeneration, No.44-1 Wenhua Road West, Jinan 250012 Shandong, China. ${ }^{3}$ Department of Human Microbiome, School and Hospital of Stomatology, Cheeloo College of Medicine, Shandong University \& Shandong Provincial Key Laboratory of Oral Tissue Regeneration \& Shandong Engineering Laboratory for Dental Materials and Oral Tissue Regeneration, No.44-1 Wenhua Road West, Jinan 250012 Shandong, China. ${ }^{4}$ Department of Oral and Maxillofacial Surgery, Peking University School and Hospital of Stomatology, 22 Zhongguancun South Avenue, Haidian, Beijing 100081, China. ${ }^{5}$ Department of Stomatology, The Second Hospital of Shandong University, No. 247 Beiyuan Road, Jinan 250033, China. ${ }^{6}$ Department of Pediatric Dentistry, School and Hospital of Stomatology, Cheeloo College of Medicine, Shandong University \& Shandong Key Laboratory of Oral Tissue Regeneration \& Shandong Engineering Laboratory for Dental Materials and Oral Tissue Regeneration, No.44-1 Wenhua Road West, Jinan 250012 Shandong, China. ${ }^{7}$ Department of Oral Medicine, Peking University School and Hospital of Stomatology, 22 Zhongguancun South Avenue, Haidian, Beijing 100081, China. ${ }^{8} \mathrm{NHC}$ Key Laboratory of Otorhinolaryngology (Shandong University), No.44-1 Wenhua Road West, Jinan, Shandong, China250012. ${ }^{9}$ These authors contributed equally: Xiaoli Ji, Hongwei Liu, Qiang Feng. ${ }^{凶}$ email: jxlbjmu@foxmail.com; hongweil5362@163.com; fengqiang@sdu.edu.cn
}

Received: 19 February 2021 Revised: 1 September 2021 Accepted: 27 September 2021

Published online: 14 October 2021 
cancer-derived MSCs can alter a significantly dysregulated secretion profile, and breast tumour cells exhibit a more aggressive phenotype through the activation of epithelial-tomesenchymal transition in vitro and in vivo [20]. To date, most related research has reported the specific cytokines secreted by tumour-associated MSCs that affect the progression of tumours, but the detailed mechanism of MSC-secreted cytokines in tumour progression is scarcely reported, although it is important to modify the TME through MSCs to prevent tumorigenesis.

In this study, we investigated the influence of OLK-MSCs (MSCs derived from OLK) and OSCC-MSCs (MSCs derived from OSCC) on OSCC cell migration and invasion through EMT. We performed RNA-sequencing to identify the key cytokines localized to specific pathways in MSCs that explain the pro-metastatic effects of OSCC-MSCs, and a neutralizing antibody, receptor inhibitor, and pathway inhibitor were used to verify the results. In addition, we also identified the upstream regulator of this pathway in MSCs, and the results were confirmed by gene overexpression, knockdown experiments, and co-immunoprecipitation.

\section{Materials and Methods}

Tumour cell line culture. OSCC cell lines CAL27(maintained in our lab) and WSU-HN6 (a kind gift from Professor Yixiang Wang at Peking University) were cultured in Dulbecco's modified Eagle's medium(DMEM,HyClone,Kansas,USA) supplemented with $10 \%$ fetal bovine serum (FBS) and 1\% penicillin-streptomycin (Gibco, Washington, USA) in a humidified incubator at $37^{\circ} \mathrm{C}$ with $5 \% \mathrm{CO}_{2}$.

Isolation of OLK-MSCS and OSCC-MSCs. Oral mucosal tissues were obtained from 16 donors (8 were pathologically confirmed as tongue OLK [21] and 8 were oral (tongue)squamous cell carcinoma [22]) at the School and Hospital of Stomatology, Cheeloo College of Medicine, Shandong University. Tissues were treated by dispase ( $2 \mathrm{mg} / \mathrm{ml}$, Sigma-Aldrich, Darmstadt, Germany) washed in PBS, and then cut into $1 \mathrm{~mm}^{3}$-sized pieces and cultured in a T25 flask with a-minimum essential medium (a-MEM; Gibco, Washington, USA) containing 10\% fetal bovine serum (FBS; Gibco, Washington, USA) and $1 \%$ penicillin-streptomycin at $37^{\circ} \mathrm{C}$ in a humidified $5 \% \mathrm{CO}_{2}$ atmosphere to obtain MSCs (passage 3-5 were used for sequent experiment). All patients signed the informed consent form. This study was approved by the ethics committee of the School and Hospital of Stomatology, Cheeloo College of Medicine, Shandong University (NO. GR201804).

\section{Identification of OLK-MSCs and OSCC-MSCs}

The methods for the identification of MSCs followed the Minimal Criteria for Defining Multipotent Mesenchymal Stromal Cells [23]. OLK-MSCs and OSCC-MSCs were treated with PE-conjugated human STRO-1, CD105 (BD Biosciences, California, USA), and CD45 and FITC-conjugated human CD29, CD73, CD90, and CD34 (BioLegend, California, USA). The control was isotype-matched control IgG or IgM. The osteogenic and adipogenic potential of OLK-MSCs and OSCC-MSCs was identified by the related induction media.

\section{Generation of conditioned medium from MSCs}

OLK-MSCs, OSCC-MSCs, CPNE7-targeting siRNA (si-CPNE7), scrambled non-targeting control siRNA (si-control), lentivirusCPNE7-GFP (Iv-CPNE7) and lentivirus-control-GFP (Iv-control) (the number of the above MSCs $=1.375 \times 10^{6}$ ) were plated at in complete medium $(13.75 \mathrm{ml})$ in a $10 \mathrm{~cm}$ plate for 5 days to generate conditioned medium (CM). The $\mathrm{CM}$ was collected through a $0.2 \mu \mathrm{m}$ filter and stored at $-80^{\circ} \mathrm{C}$.

\section{Transwell migration and invasion assay}

These assays were measured in 24-well Transwell inserts (24well, $8.0 \mu \mathrm{m}$, Corning, NY, USA) with or without Matrigel $(300 \mu \mathrm{g} / \mathrm{ml}$, Corning, NY, USA). OLK-MSCs and OSCC-MSCs $\left(5 \times 10^{4}\right.$ for 5 days),
CM derived from OLK-MSCs or OSCC-MSCs, serum-free CM derived from OLK-MSCs or OSCC-MSCs (added 10\% FBS), CM of OSCCMSCs alone or in the presence of a human CXCL8 neutralizing antibody $(0.4 \mu \mathrm{g} / \mathrm{ml}$, R\&D Systems, Minnesota, USA) (or control lgG (negative control, Santa-Cruz, California, USA)), medium added with $2000 \mathrm{pg} / \mathrm{ml}$ and $30000 \mathrm{pg} / \mathrm{ml}$ CXCL8 (PeproTech $\mathrm{GmbH}$, Hamburg, Germany), CM from si-CPNE7 and si-control or CM from Iv-CPNE7 and Iv-control (the volume of $\mathrm{CM}=500 \mathrm{ul}$ ) were added to the lower chamber ( 24 well plate). CAL27 cells $\left(10^{5}\right)$ or WSUHN6 cells $\left(5 \times 10^{4}\right)$ (or treated with $400 \mathrm{nM} \mathrm{CXCR2} \mathrm{inhibitor}$ SB225002 (TargetMol, Boston, MA, USA) or $0.001 \%$ DMSO) in serum-free medium were added to the upper Transwell inserts (24well, $8.0 \mu \mathrm{m}$, Corning, NY, USA). After incubation at $37^{\circ} \mathrm{C}$ for $18 \mathrm{~h}$, a cotton swab was used to remove the cells of the upper chamber and the invaded cancer cell on the lower side of the membrane were fixed with $4 \%$ paraformaldehyde and stained with $0.1 \%$ crystal violet for $30 \mathrm{~min}$, respectively. The number of invaded cancer cells on the lower side of the membrane was counted with a light microscope in three randomly selected fields at $100 \times$ and $400 \times$ magnification. The average number of each field was calculated.

\section{Animals' models}

Four-week-old female BALB/c nude mice (SPF(Beijing)BIOTECHNOLOGY Co., Ltd.) were maintained in a sterile environment. $10^{6}$ OLK-MSCs $+10^{6}$ WSU-HN6 or $10^{6}$ OSCC-MSCs $+10^{6}$ WSU-HN6 in PBS injected into the tail vein of BALB/C nude mice. All mice were killed by 4 weeks after injection. The metastatic nodules in the lung were recorded and stained with H\&E. The animal experiments were approved by the ethics committee of the School and Hospital of Stomatology, Cheeloo College of Medicine, Shandong University (NO. GR201804).

\section{Western blot assay}

Protein was obtained with RIPA buffer. Equal amounts of proteins were resolved by $10 \%$ SDS-PAGE [24] and immunoblotted with primary antibodies against the following antigens overnight: E-cadherin, alpha E catenin, Vimentin, N-cadherin, GFP (Proteintech, Wuhan, China), p-p65, total-p65 (Abcam, Cambridge, England), p-Ікßa, total-Iкßa (CST, California, USA), CPNE7 (GeneTex, Texas, USA), and GAPDH (Affinity, Ohio, USA), incubated with the HRP-conjugated secondary antibodies (Affinity, Ohio, USA). Positive immunoreactive bands were detected by enhanced chemiluminescence (ECL, Amersham Pharmacia Biotech, USA) reaction and normalized by GAPDH with quantity one.

\section{RNA-sequencing analysis}

Total RNA was extracted from OLK-MSCs and OSCC-MSCs (3 cases in each group) with Trizol (Thermo Fisher Scientific) and total RNA was isolated according to the manufacturer's protocol. The differentially expressed genes were identified with edgeR (3.30.3) $(p<0.05$, | fold change(FC) $>2)$, and KEGG pathway analysis was performed (Database string $C$ ).

\section{Retrieval of The Cancer Genome Atlas (TCGA) data}

TCGA expression data were retrieved from UALCAN (http://ualcan. path.uab.edu/). TCGA datasets were stratified into normal and primary tumours.

\section{Enzyme-linked immunosorbent assay (ELISA)}

The CM of OLK-MSCs, OSCC-MSCs, si-CPNE7, si-control, Iv-CPNE7, and Iv-control was obtained as described above. The concentration of CXCL8 was measured by ELISA (BioLegend, California, USA) according to the manufacturer's instructions.

\section{Immunohistochemistry (IHC) assays}

Immunohistochemical staining was routinely performed according to a standard protocol. The sample (42 OLK, 62 OSCC) were 
obtained from the Department of Oral Pathology (School and Hospital of Stomatology, Cheeloo College of Medicine, Shandong University). Histopathological evaluation of samples was performed by two pathologists. Samples were stained with a CPNE7 antibody (Bioss, Beijing, China) [25]. For the immunohistochemical scoring of CPNE7, the staining CPNE7-intensity was scored as 0 (negative), 1 (low Positive), 2 (positive), and 3 (high positive) [26] and the proportions of CPNE7-positive cells were counted using the Fiji open-source image processing program (ImageJ software, USA) [26]. Both the CPNE7-staining intensity and the proportions of CPNE7-positive cells were taken as the average value of three random optical fields in the epithelial and connective tissue, respectively $[26,27]$. The multiplication for the above intensity and proportion was used to evaluate the expression level of CPNE7 $[26,27]$.

\section{Immunofluorescence histochemistry}

Tissues collected from patients were stored at $-80^{\circ} \mathrm{C}$ and cut into $4 \mu \mathrm{m}$ sections. Alternatively, MSCs were seeded in 12 -well plates with sections overnight and fixed with $4 \%$ paraformaldehyde. Sections were incubated with $3.0 \%$ hydrogen peroxide and then blocked in $5.0 \%$ bovine serum albumin. The sections were incubated at $4{ }^{\circ} \mathrm{C}$ overnight with antibodies against CPNE7 (Bioss, Beijing, China), STRO-1 (Santa -Cruz, California, USA), or p65 (CST, California, USA), Nucleolin. Subsequently, incubation with Alexa Fluor 488/594-labelled goat anti-mouse/rabbit IgG (Abbkine, California, USA) was for $1 \mathrm{~h}$ at $37^{\circ} \mathrm{C}$ in the dark.

\section{Small interfering RNA (siRNA) transfection}

si-CPNE7 and si-control were obtained from GenePharma (Suzhou, China). The sequences were as follows: CPNE7 sense $5^{\prime}-\mathrm{CC}$ GGGAAAGCCUCUCAAUATT-3', CPNE7 antisense 5'-UAUUGAGAGGC UUUCCCGGTT-3', control sense 5'-UUCUCCGAACGUACGUTT-3', and control antisense 5'-ACGUGACACGUUCGGAGAATT-3'. $2 \times 10^{5}$ cells were seeded in each well of a 6-well plate overnight. $10 \mathrm{nM}$ siRNA was diluted in Opti-MEM ${ }^{\oplus}$ I Reduced Serum Medium without serum and mixed with Lipofectamine 2000 RNAiMAX Reagent (Thermo Fisher Scientific, New York, USA). The mixture was added to each well and incubated for $6 \mathrm{~h}$.

\section{Lentivirus and transfection}

The Iv-CPNE7 and Iv-control were constructed by Gene Pharma (Suzhou, China). OLK-MSCs were incubated with a medium containing lentivirus and polybrene. After $24 \mathrm{~h}$, the medium was changed, and incubation proceeded for $48 \mathrm{~h}$.

\section{Co-IP assay}

Iv-CPNE7 transfected OLK-MSCs' lysates were treated by NP-40 buffer. The lysates were incubated at $4{ }^{\circ} \mathrm{C}$ overnight with GFP or total total-IKßa, Nucleolin and normal IgG (Santa, California, USA) with rotation. Protein A/G PLUS agarose beads (Santa, California, USA) were added and incubated at $4{ }^{\circ} \mathrm{C}$ for $1 \mathrm{~h}$ with rotation. The complexes were released from the beads by boiling in an SDSPAGE sample buffer. The immunoprecipitates were analysed by western blotting with GFP, CPNE7, and total-Iкßa, Nucleolin.

\section{Statistical analysis}

All experiments were repeated 3 times and the data are presented as mean $\pm S D$. Comparison between two groups were performed using paired and two-tailed Student's $t$-test. The considered statistically significant was $p \leq 0.05$. The data were analysed using SPSS software version 17.0.

\section{RESULTS}

\section{Identification of OLK-MSCs and OSCC-MSCs}

To verify the characteristics of MSCs, a panel of antibodies against MSC surface antigens was used to label OLK-MSCs and OSCC-MSCs and analyse the cells by flow cytometry. The results showed that OLK-MSCs and OSCC-MSCs were positive for CD29, CD73, CD90, CD105, and STRO-1 and negative for CD34 and CD45 (Supplementary Fig. 1A). Multilineage differentiation experiments showed that OLK-MSCs and OSCC-MSCs could form mineralized nodules under osteoinductive conditions (Supplementary Fig. 1B), form oil droplets upon adipogenic induction (Supplementary Fig. 1C), and chondrogenesis (Supplementary Fig. 1D), respectively.

\section{OSCC-MSCs promote cancer metastasis by EMT}

To evaluate the effect of OLK-MSCs and OSCC-MSCs on the migration and invasion of OSCC cells, we constructed a two-cell co-culture system using a transwell chamber and showed that coculture with OSCC-MSCs significantly increased the number of migrated and invasive CAL27 and WSU-HN6 cells $(p<0.05)$ compared with OLK-MSC co-culture (Fig. 1A). To further verify the metastasis-promoting effect of OSCC-MSCs, CM from OLKMSCs and OSCC-MSCs was obtained and used to treat CAL27 and WSU-HN6 cells in transwell assays. The results showed that the migration capability of CAL27 and WSU-HN6 cells was enhanced by OSCC-MSC-CM compared to OLK-MSC-CM $(p<0.05)$ (Fig. 1B). Furthermore, the metastasis-promoting effect of OSCC-MSC-CM was like that observed in the OSCC-MSC-cancer cell co-culture system. To eliminate the effect of serum consumption during generation of conditioned medium, CM was acquired in no FBS medium of OLK-MSCs and OSCC-MSCs for 5 days. The results showed that pro-metastasis of OSCC-MSCs was also confirmed and not due to the serum deprivation (Supplementary Fig. 2).

We next tested whether OSCC-MSCs can effectively promote OSCC cell in vivo, $10^{6}$ OLK-MSCs and $10^{6}$ WSU-HN6 or $10^{6}$ OSCCMSCs and $10^{6}$ WSU-HN6 were injected via the tail vein of nude mice. Fig $1 \mathrm{C}$ indicated that typical cancer tissues changes within the lungs staining with $\mathrm{H} \& \mathrm{E}$ in two groups and the group of OSCC-MSCS + WSU-HN6 revealed a remarkable increase in the number of metastatic nodules in the lung when compared with OLK-MSCs $(p<0.05)$. So, the pro-metastatic effect of OSCC-MSCs was be showed.

The expression of E-cadherin, alpha E catenin (epithelial markers), N-cadherin, and Vimentin (epithelial markers) was shown that the levels of E-cadherin and alpha $E$ catenin decreased significantly in CAL27 and WSU-HN6 cells treated with OSCC-MSC-CM compared to the OLK-MSC-CM group $(p<0.05)$, while the expression of $\mathrm{N}$-cadherin notably increased when CAL27 and WSU-HN6 cells were treated with OSCC-MSC-CM in comparison with OLK-MSC-CM $(p<0.05) \quad$ (Fig. 1D, E). No differences in Vimentin were detected between the two groups $(p>0.05)$ (Fig. $1 \mathrm{D}, \mathrm{E})$.

\section{OLK-MSCs and OSCC-MSCs display different gene expression profiles}

To explore the cytokines involved in OSCC cell metastasis, RNAsequencing (SRA data (PRJNA665945)) was conducted and showed 454 differentially expressed genes $\left(\left|\log _{2} \mathrm{FC}\right|>1, p<\right.$ 0.05):247 genes were upregulated and 207 downregulated in OSCC-MSCs when compared with OSCC-MSCs (Fig. 2A). Figure 2B showed the fold change of the differential genes secreted by MSCs were related to metastasis. And fold change of secreted CXCL8-chemokines was the greatest (upregulated significantly) [27-30]. KEGG analysis showed that several pathways associated with cancer metastasis were activated $(p<0.05)$ (Fig. 2C). CXCL8 has also annotated the above pathway especially the IL-17 signaling pathway, NOD-like receptor signaling pathway, NF-KB pathway, Phospholipase D signaling pathway (Fig. 2C) [31-33]. NF-KB pathway (BIRC3, CARD10, CD14, CXCL8, PTGS2, TNFAIP3) is the centre of the above pathway $[3,34,35]$. Additionally, TCGA data showed that CPNE7 expression in primary tumours was significantly upregulated compared to that in normal tissues in Cervical squamous cell carcinoma (CESC) and Head and Neck 

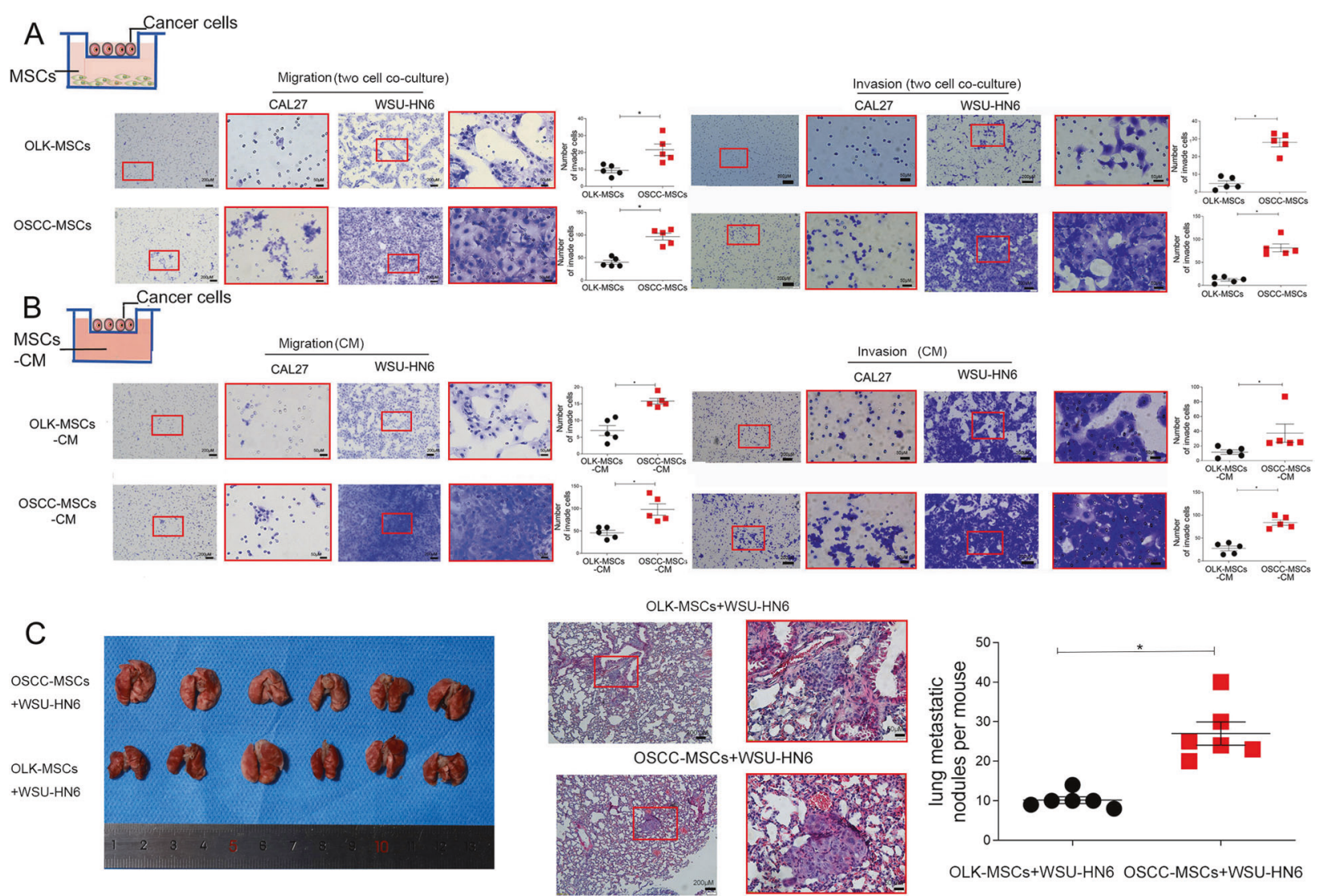

D
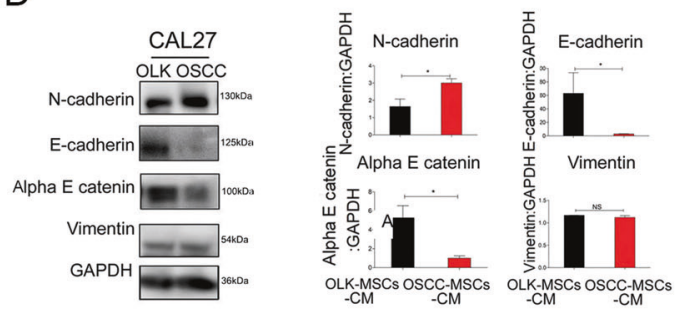

$\mathrm{E}$
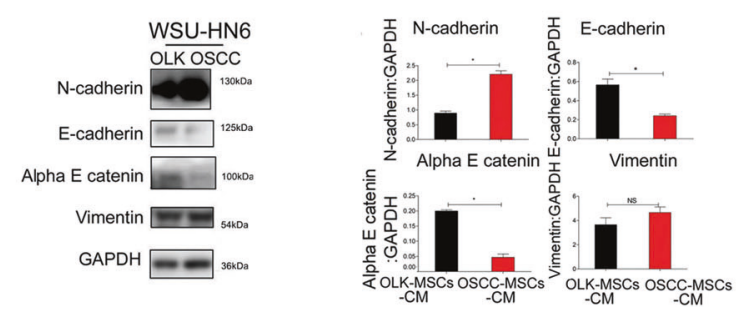

Fig. 1 OSCC-MSCs promoted the metastasis of OSCC cell lines. Transwell assays were performed to detect tumour (CAL27 and WSU-HN6) cell migration and invasion through indirect cell-cell contact co-culture (A) and conditioned medium (B) from OLK-MSCs and OSCC-MSCs $(n=$ 5). C OLK-MSCs or OSCC-MSCs $\left(10^{6}\right)$ were co-injected WSU-HN6 $\left(10^{6}\right)$ into BALB/c mice via tail vein $(n=6)$. Metastasis lung tumor nodules were counted after 28 days. OSCC-MSCs promote the metastasis of CAL27 (C) and WSU-HN6 cells (D) through epithelial-mesenchymal transition (EMT)-related proteins (E-cadherin, $\mathrm{N}$-cadherin, alpha $\mathrm{E}$ catenin, and Vimentin). The tests were repeated three times. Data were expressed as means \pm SD. ${ }^{*} p<0.05$.

squamous cell carcinoma (HNSC) $(p \leq 0.05)$ (Fig. 2D) and could promote nodal metastasis (Fig. 2E). According to the data of RNAsequencing analysis, CPNE7 in MSCs (the upregulated differential gene) (Fig. 2A) was predicted as a potential regulator of the NF-KB pathway [36] to promote the metastasis of tumour cells.

\section{OSCC-MSCs secrete more CXCL8, which is regulated by NF-KB pathways}

To validated the results of RNA-sequencing, ELISA showed that compared to OLK-MSCs, secreted CXCL8 was also significantly elevated in OSCC-MSCs $(n=5, p<0.05)$ (Fig. 3A). Additionally, to further determine the metastasis-promoting effect of CXCL8 on OSCC cells, we inhibited the effect of CXCL8 with a neutralizing anti-CXCL8 antibody and an inhibitor of the CXCL8 receptor CXCR2 (SB225002). The results showed that the metastatic effect of OSCC-MSC-CM was significantly inhibited (Fig. 3B, 3C), while $\mathrm{E}$-cadherin expression increased and $\mathrm{N}$-cadherin expression in CAL27 and WSU-HN6 cells decreased (Fig. 3B, 3C). Additionally, to stimulate the microenvironments of OLK-MSCs and OSCCMSCs, different concentrations of CXCL8 $(2000 \mathrm{pg} / \mathrm{ml}$ and $30,000 \mathrm{pg} / \mathrm{ml}$ ) were used by migration and invasion assay. The results were like CXCL8 blockade or CXCR2 inhibitor assay (Supplementary Fig. 3).

To confirm the regulation of NF-KB on CXCL8 secretion, p65 was released and translocated into the nucleus in OSCC-MSCS and only found in the cytoplasm of OLK-MSCs by the immunofluorescence assay which confirm NF-KB pathway activation (Fig. 3D). In order to further support the above conclusion, the additional Western blotting analysis showed that phosphorylated p65 levels were increased in OSCC-MSCs of the different individuals $(n=5)$ compared with OLK-MSCs $(n=5) \quad(p<0.05)$ (Fig. 3E). Furthermore, phosphorylated $1 \mathrm{KBa}$ in the cytoplasm, leading to its ubiquitination and subsequent degradation, could promote $\mathrm{p} 65$ is released, translocate to the nucleus, and regulate the expression of genes $[37,38]$. Therefore, we detected that OSCC-MSCs $(n=5)$ expressed significantly higher level of 
A
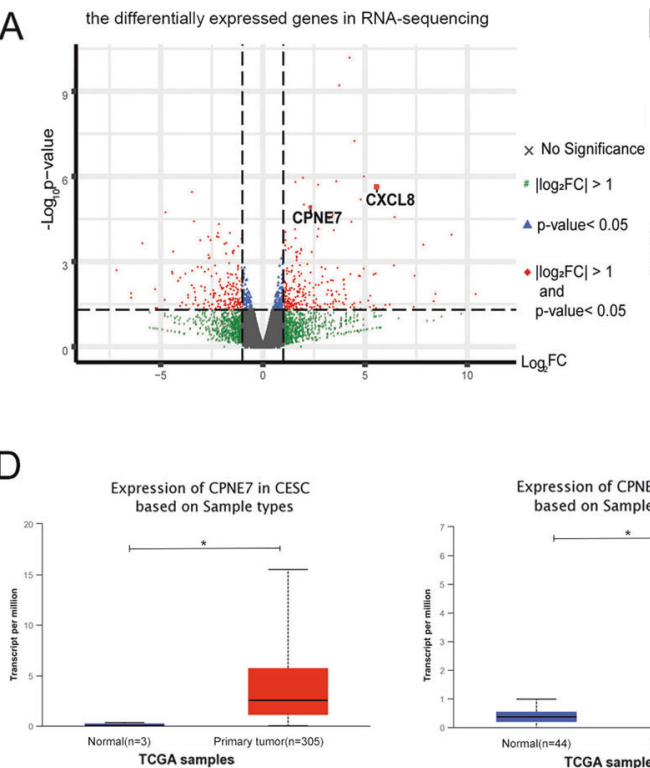

$\mathrm{B}$ the secreted cytokines on metastasis

in RNA-sequencing
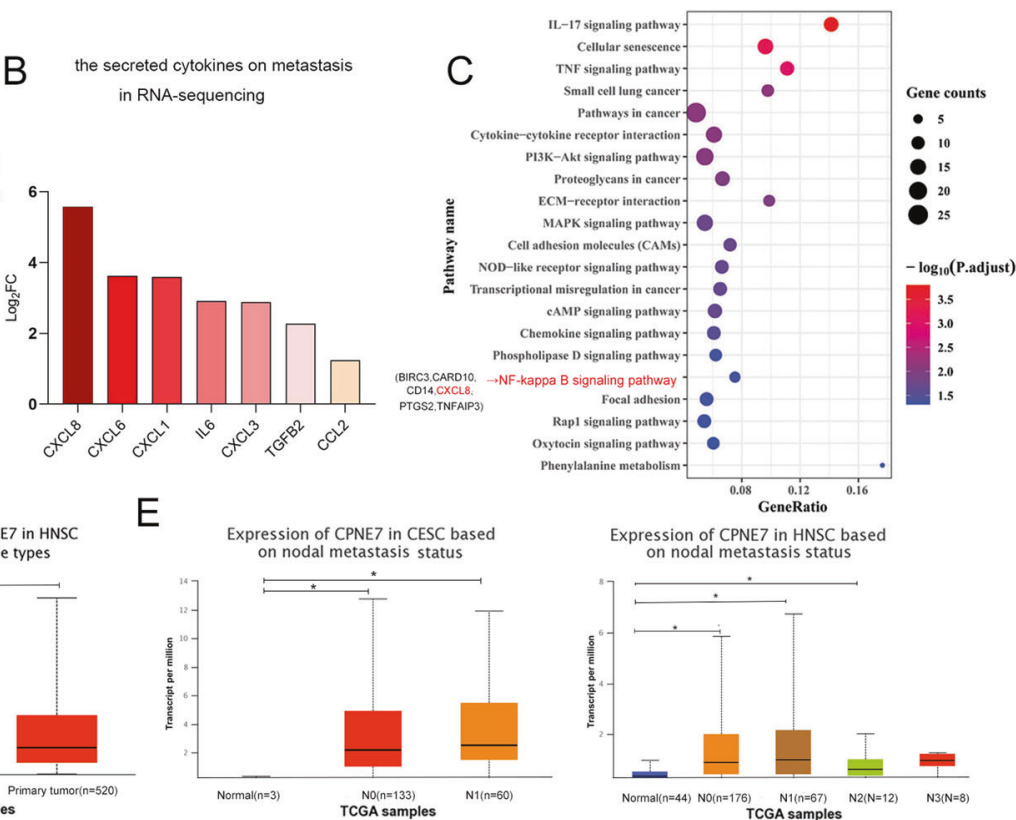

$E$
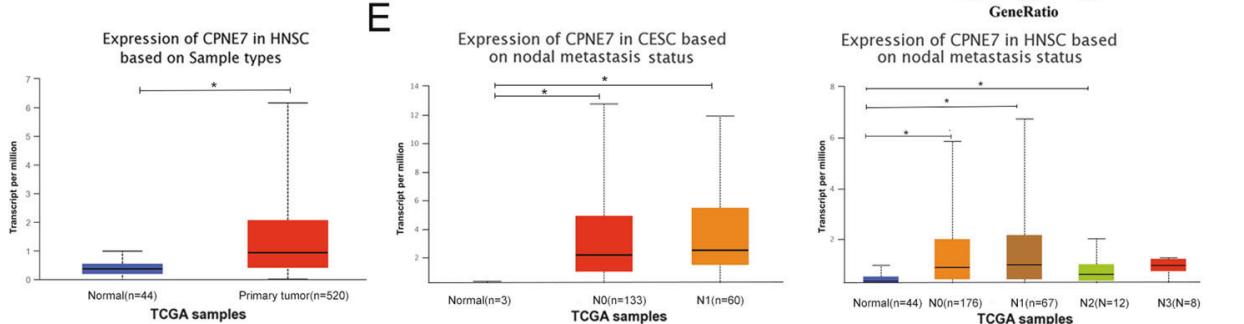

Fig. 2 CPNE7 regulates CXCL8 secretion through NF-KB pathway in RNA-sequencing analysis. A volcano plot presenting all of the

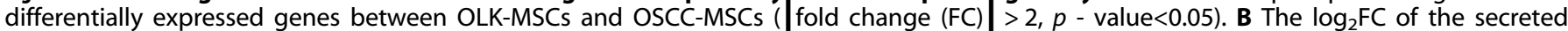
cytokines on metastasis. C KEGG pathway analysis between OLK-MSCs and OSCC-MSCs $(p<0.05)$. D Expression of CPNE7 in Cervical squamous cell carcinoma (CESC) and Head and Neck squamous cell carcinoma (HNSC) according to TCGA database. E Expression of CPNE7 in CESC and HNSC based on nodal metastasis status. N0: No regional lymph node metastasis. N1: Metastases in 1 to 3 axillary lymph nodes. N2: Metastases in 4 to 9 axillary lymph nodes. N3: Metastases in 10 or more axillary lymph nodes. ${ }^{*} p<0.05$.

phosphorylated IKßa levels than OLK-MSCs $(n=5) \quad(p<0.05)$ (Fig. 3F). For the further demonstration of the NF-KB pathway regulation of CXCL8, OSCC-MSCs were treated with NF-KB inhibitor (BAY 11-117802), and results demonstrated that CXCL8 secretion was partially reduced (Fig. 3G).

\section{CPNE7 is upregulated in OSCC tissue and OSCC-MSCs}

CPNE7 was predicted as a potential regulator of the NF-KB pathway by KEGG pathway annotation and RNA-sequencing analysis [36]. To explore the expression level of CPNE7, we performed immunohistochemically staining in clinical tissue. As shown in Fig. 4A, the expression of CPNE7 was detected in the nucleus and cytoplasm and was significantly upregulated in the total tissue or stromal tissue of OSCC compared to OLK. Moreover, immunofluorescent staining showed that CPNE7 and STRO-1 were co-expressed in stromal tissue and MSCs (OLK and OSCC) (Fig. 4B). In addition, we observed an increase in CPNE7 expression in OSCC-MSCs compared with OLK-MSCs (Fig. 4C).

\section{Upregulation of CPNE7 in OSCC-MSCs promote cancer metastasis by EMT}

To verify the regulatory effect of CPNE7 on the NF-KB pathway, we performed an MSC transfection assay to overexpress and knockdown CPNE7. We used siRNA to knockdown CPNE7 expression in OSCC-MSCs, and the treatment inhibited CAL27 and WSU-HN6 cell metastasis (Fig. 5A). In addition, the increase in E-cadherin and decrease in $\mathrm{N}$-cadherin expression were determined by western blotting when CM was acquired from si-CPNE7-treated OSCCMSCs co-cultured with CAL27 or WSU-HN6 (Fig. 5A).

A lentivirus was used to overexpress CPNE7 (Iv-CPNE7) in OLKMSCs. Transwell assays showed that CM obtained from OLK-MSCs treated with Iv-CPNE7 significantly increased the metastatic ability of CAL27 and WSU-HN6 cells (Fig. 5B). Western blotting assays showed that the expression of $\mathrm{N}$-cadherin was increased, with a simultaneous decrease in the expression of E-cadherin by $\mathrm{CM}$ of OLK-MSCs treated with Iv-CPNE7 (Fig. 5B).

\section{CPNE7 interacts with Nucleolin,Iкßa and activeate NF-кB signalling pathway}

siRNA was used to knock down CPNE7 expression in OSCC-MSCs, CXCL8 level was significantly decreased in OSCC-MSCs treated with si-CPNE7 (Fig. 6A), and phosphorylated p65 and IKßa decreased compared with those in the control (Fig. 6C).

Compared with Iv-control, ELISA assay was used to measure the abundance of CXCL8 in the two groups, and the results showed that OLK-MSCs overexpressing CPNE7 (IV-CPNE7) could secrete more CXCL8 than the control cells (Fig. 6A). Immunofluorescence assay was performed showed that compared with IV-control, in the lentivirus-CPNE7-GFP (Iv-CPNE7) the expression of p65 and GFP could was found in cytoplasm and nucleus. So, it speculated that CPNE7 could promote p65 released and translocated into the nucleus in OSCC-MSCS and p65 may be active in the NF-KB pathway (Fig. 6B). Furthermore, p65 and IKßa phosphorylation were also increased in OLK-MSCs treated with Iv-CPNE7 (Fig. 6C).

To determine the possible mechanisms of CPNE7 in the regulation of the NF-KB pathway, we predicted an interaction between CPNE7 and IKßa. To confirm this speculation, a Co-IP assay was performed in OLK-MSCs treated with Iv-CPNE7. In CPNE7overexpressing OLK-MSCs, CPNE7 was fused with GFP protein. The cell lysate was incubated with lgG beads, Nucleolin, GFP, and IKßa antibodies and the immunoprecipitated proteins were obtained. The two kinds of lysate assays showed that Nucleolin - GFP (CPNE7)- IKßa could be pulled down (Figs. 6D, 6F). The above results suggest that CPNE7 could directly bind to IKßa, Nucleolin.

In addition, we performed an immunofluorescence assay of tissue and MSCs with Nucleolin and CPNE7. The results showed that CPNE7 protein co-localized with Nucleolin protein in the cytomembrane (cytoplasm and nucleus) of stromal tissue and MSCs (Fig. 6E). Next, we investigated the changing pattern of CPNE7 and Nucleolin in the progression from OLK-MSCs to OSCCMSCs. Western blotting assays showed an increase in CPNE7 expression, whereas the expression of Nucleolin remained unchanged (Supplementary Fig. 4A). Consistent with the above results, no changes in Nucleolin were detected in OSCC-MSCs or 
A

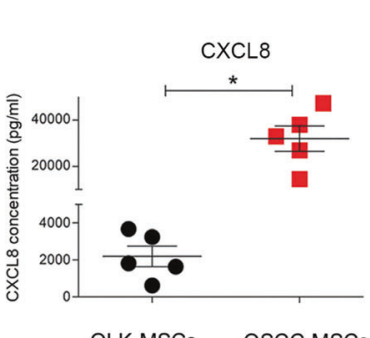

OLK-MSCs
$\mathrm{B}$

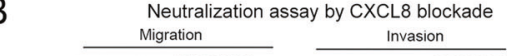
Invasion

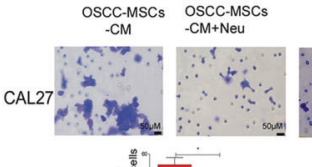
$\begin{array}{cc}\underset{\text { OSCC-MSCS }}{\text { OSCC-MS }} & \text {-CM+Neu } \\ -\mathrm{CM} & \end{array}$
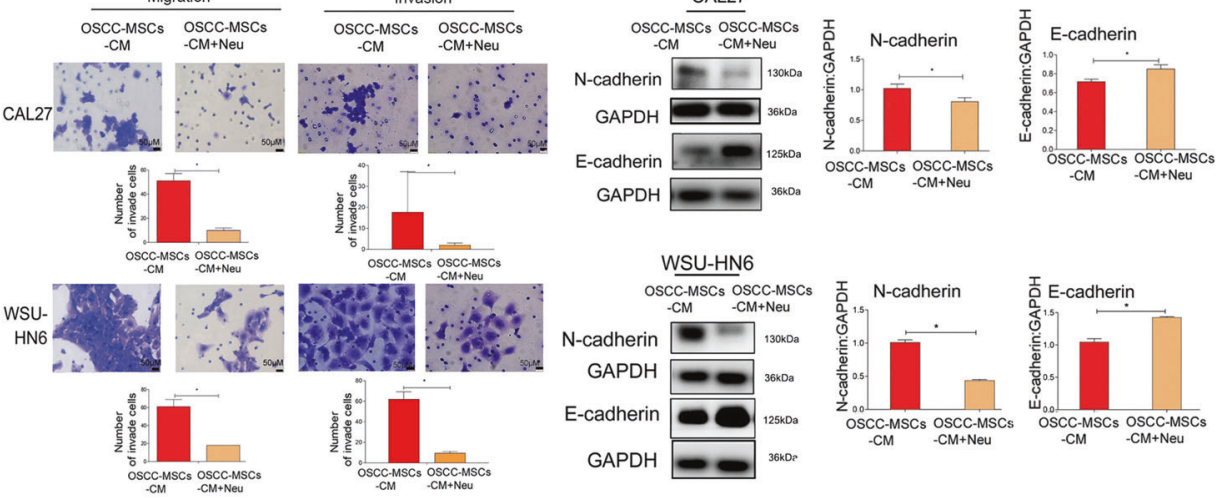

E-cadherin

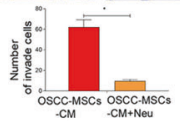

CXCR2 inhibitor assay

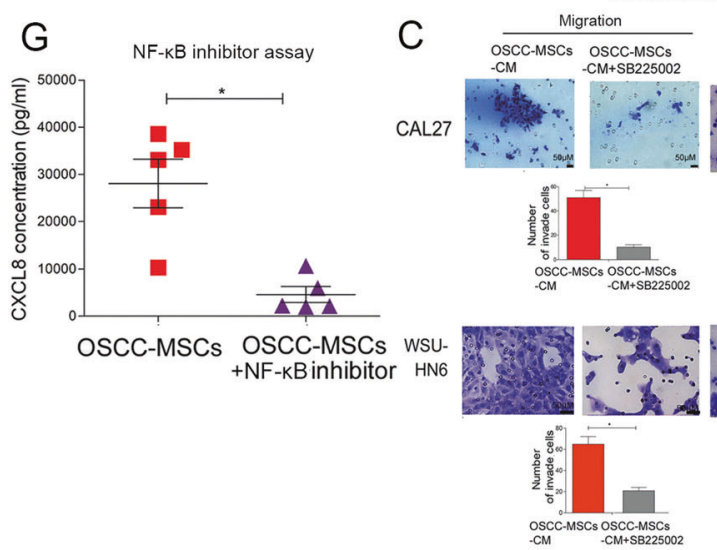

OLK-MSCs
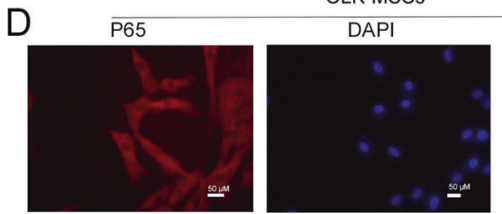

OSCC-MSCS

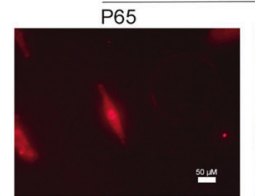

DAPI

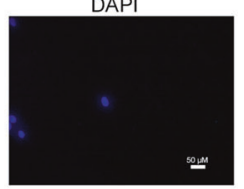

Merge

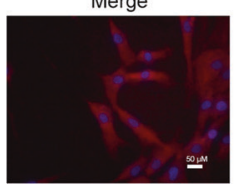

Merge

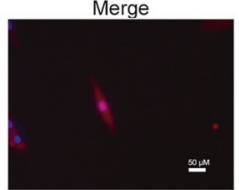

\begin{abstract}
ay
\end{abstract}
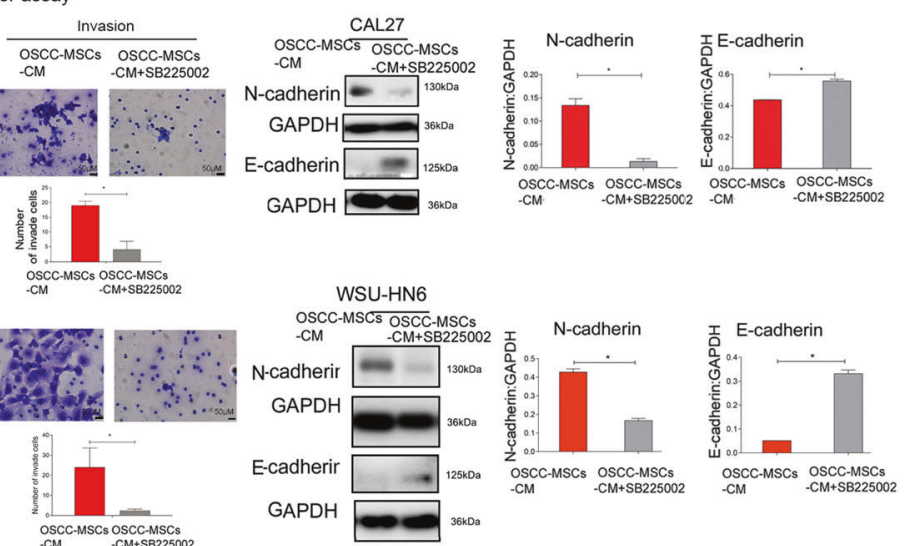

E
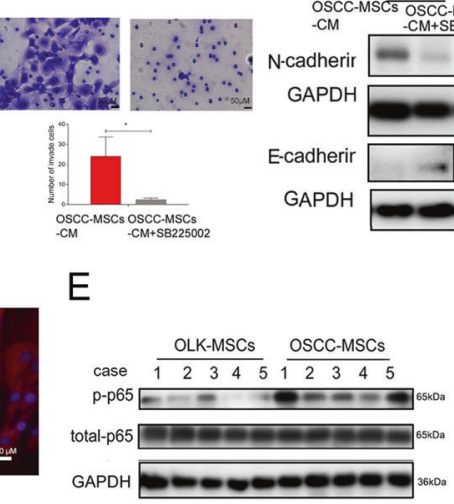

$\mathrm{F}$
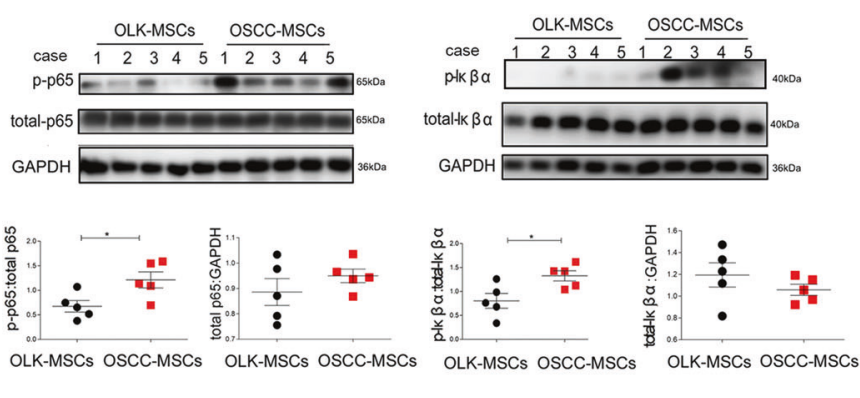

Fig. 3 Overexpression CXCL8 secretion regulated by NF-kB pathway in OSCC-MSCs. A The level of secreted CXCL8 was observed in the conditioned medium of OLK-MSCs and OSCC-MSCs $(n=5)$. Neutralizing antibody (CXCL8) (B) and CXCR2 inhibitor (SB225002) (C) inhibited OSCC-CM-induced OSCC cell line metastasis through EMT -related proteins. D Representative immunofluorescence images of the translocation of p65 in OLK-MSCs and OSCC-MSCs. Scale bars $=50 \mu \mathrm{m}$. Western blotting was used to measure the differential expression of

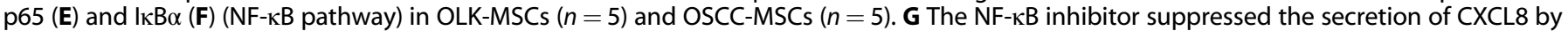
Elisa. The tests were repeated three times. Data were expressed as means \pm SD. ${ }^{*} p<0.05$.

OLK-MSCs treated with si-CPNE7 (Supplementary Fig. 4B, C). It speculated that Nucleolin may be the surface recpotor of CPNE7 [39], and combined with IKßa to active NF-KB signalling pathway when they move into the cytoplasm.

\section{DISCUSSION}

The ability of MSCs to reprogram the TME plays a crucial role in cancer initiation and progression, and the existence of paracrine loops between MSCs and tumour cells has been suggested [10]. However, few studies have examined the role of MSCs in the development of OLK into OSCC. Here, we report that compared with OLK-MSCs, OSCC-MSCs can promote OSCC cell migration through EMT. In addition, CPNE7 bind to Nucleolin in cell surface and then conjugates with IKßa when CPNE7 moved into cytoplasm. They could promote IKßa phosphorylation and p65 translocate into nucleus activated the NF-KB pathway, which is closely associated with the metastasis of OSCC cells through CXCL8 secretion (Fig. 7).

Herein, this study showed that CAL27 and WSU-HN6 cells possessed stronger metastatic ability regulated by EMT when treated with OSCC-MSCs than OLK-MSCs. Similar results were shown not only in cell-cell indirect contact but also in the CM treatment method via the transwell system, which agreed with previous studies that tumour-derived (TME educated) MSCs could promote the metastasis of tumours through EMT [20,40]. In the TME, the interaction between MSCs and tumours is indirect contact through soluble secreted factors $[9,10]$. We observed that 
A
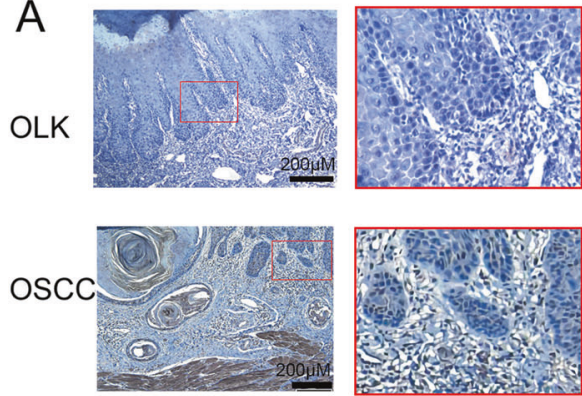

$\mathrm{B}$
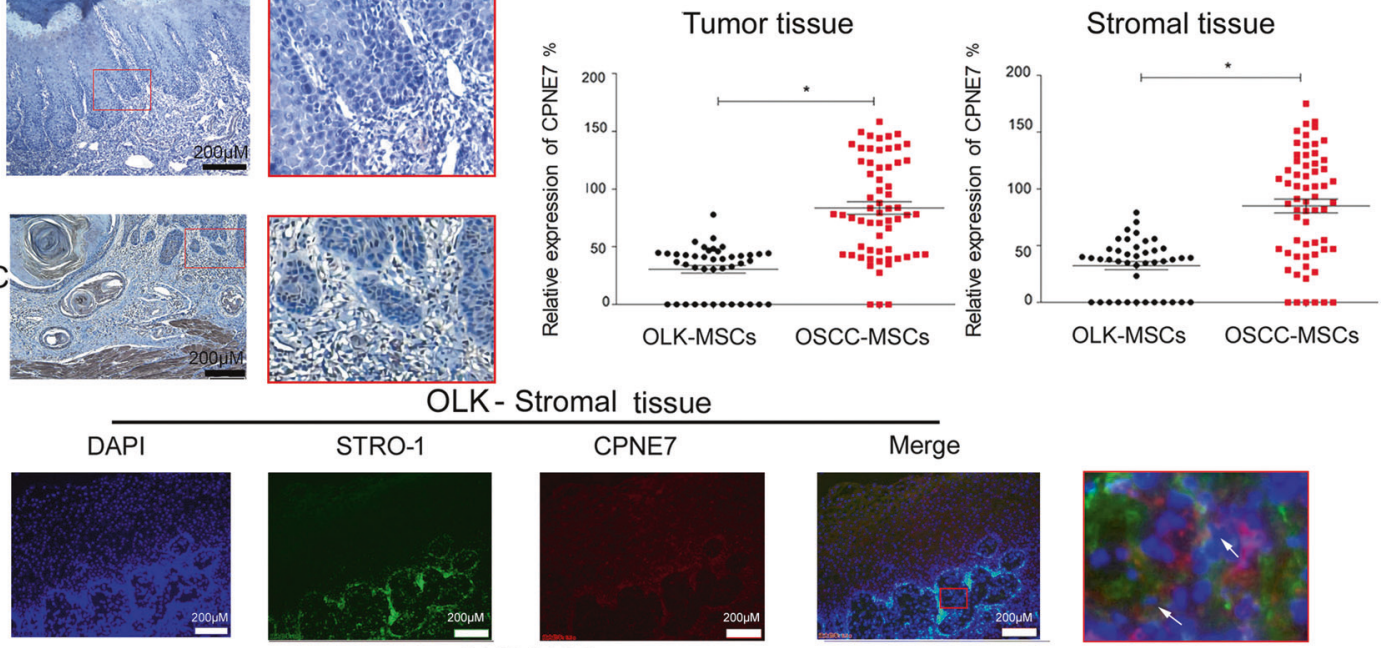

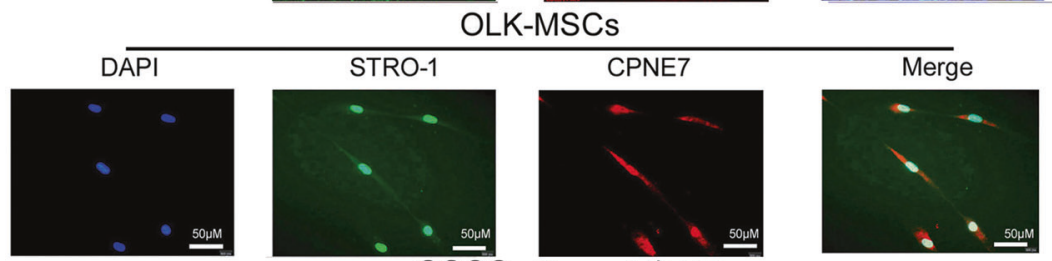

OSCC - Stromal tissue
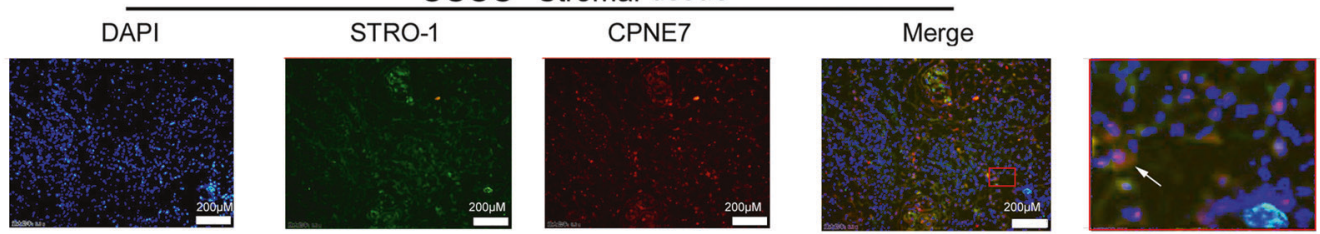

OSCC-MSCs

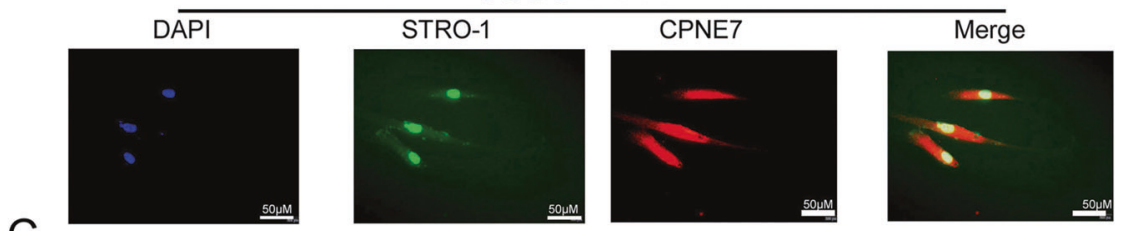

C
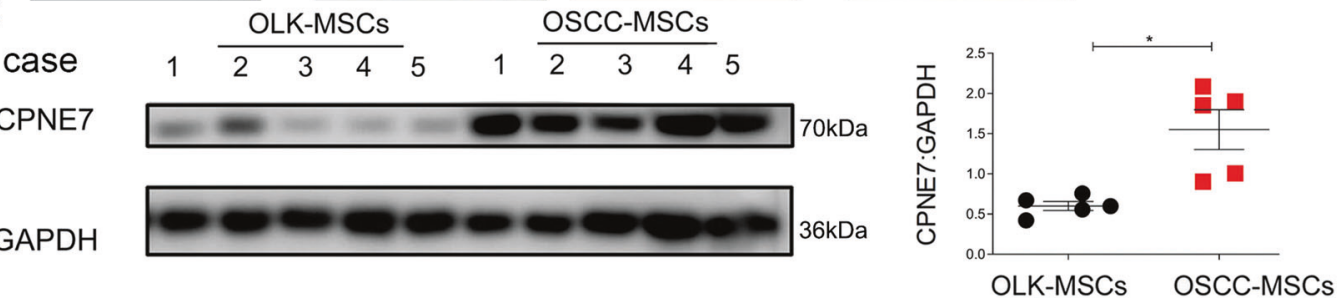

Fig. 4 Higher expression of CPNE7 in tissue and MSCs of OSCC than OLK. A Representative immunohistochemical images of CPNE7 expression levels in OLK and OSCC tissues. B Immunofluorescence detection of the expression of CPNE7 and the MSC marker STRO-1 in tissue and MSCs. The arrow indicated the co-expression of CPNE and STRO-1 in the same cell of tissue. C Western blotting was performed to detect the expression of CPNE7 between OLK-MSCs and OSCC-MSCs $(n=5)$. The tests were repeated three times. Data were expressed as means \pm SD. ${ }^{*} p<0.05$.

more CXCL8 was expressed and secreted by OSCC-MSCs to increase the metastatic ability of OSCC, which was also confirmed by the CXCL8 neutralizing antibody and CXCR2 inhibitor experiments. A study reported that CXCL8, a pro-inflammatory chemokine, plays an important role in metastasis by associating with tumour-related inflammation [27-30] and inducing the risk of OLK and OSCC, such as in the case of betel quid chewing and smoking $[41,42]$, but the regulatory pathways are still not reported to date. In our study, KEGG pathway analysis showed that CXCL8 was enriched in the nuclear factor-KB (NF-KB), the centre of some annotated signalling pathway [3], is essential for the initiation and maintenance of EMT [43]. Additionally, NF-KB signalling was active and the pathway inhibitor partially reduced the production of CXCL8 in the CM of OSCC-MSCs. Previous findings showing that tumour-derived MSCs secreted CXCL8 regulated by the NF-KB pathway were also confirmed in other tumours [31-33], and we extended these observations further by identifying the upstream regulator.

To study the regulator of the NF-KB pathway, RNA-sequencing was performed, which identified CPNE7 [36] associate with nodal metastasis status in the TCGA database. CPNE7, derived from the epithelium, is a ubiquitous calcium-dependent 

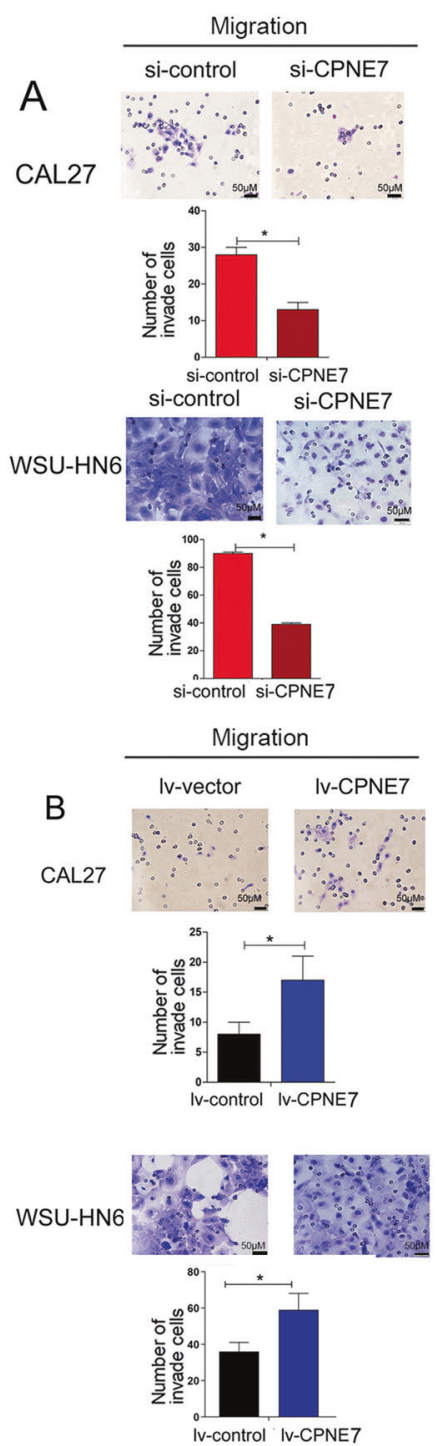
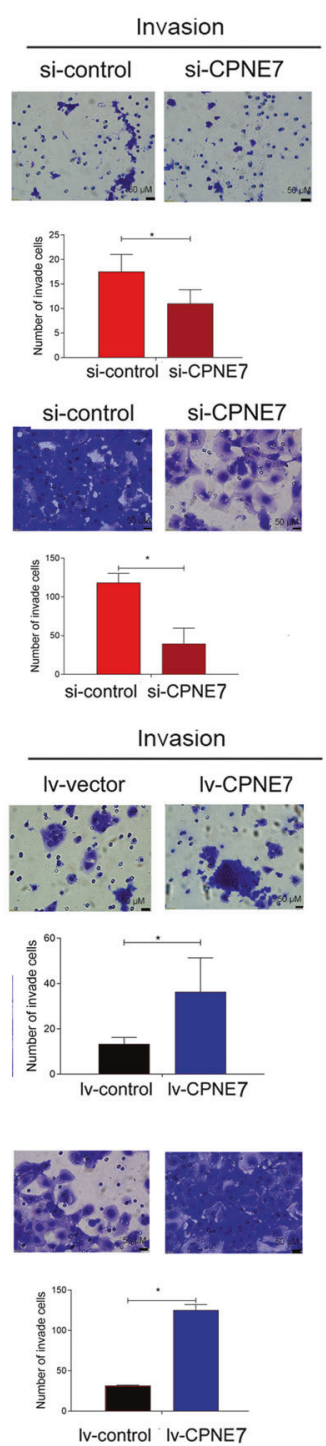
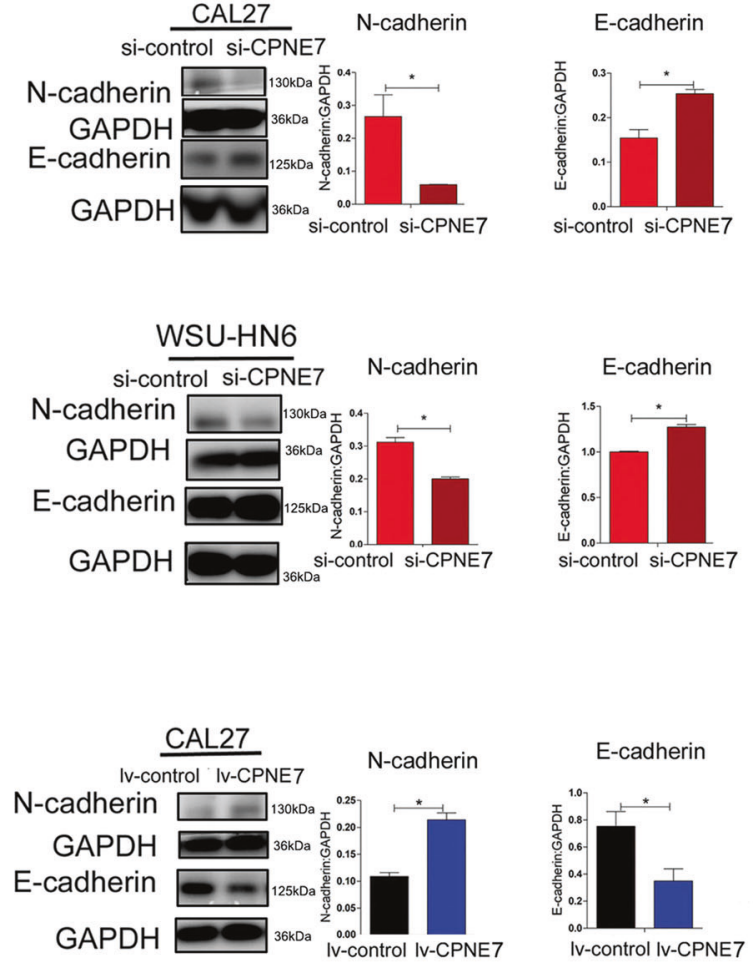

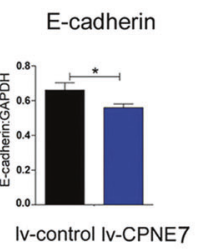

Fig. 5 Overexpression and knockdown of CPNE7 in MSCs verified that CPNE7 promote metastasis of OSCC cell lines. A Knockdown of CPNE7 in OSCC-MSCs suppressed the migration of OSCC cell lines through EMT. B Overexpression of CPNE7 in OLK-MSCS promoted the migration of OSCC cell lines through EMT. The tests were repeated three times. Data were expressed as means \pm SD. ${ }^{*} p<0.05$.

phospholipid-binding protein [44, 45]. First, our results showed that the expression of CPNE7 was increased in the connective tissue and MSCs of OSCC compared to OLK. CPNE7 was coexpressed with STRO-1 (one of the markers of MSCs $[46,47]$ ) in the connective tissue of OLK and OSCC. Our results on the CPNE7 expression level were partly confirmed by tumour tissue data from the TCGA database (in comparisons between paraand tumour tissues). Second, we knocked down and overexpressed CPNE7 in MSCs and found that the CM could affect metastasis and $\mathrm{E}$-cadherin and $\mathrm{N}$-cadherin expression regulated by CXCL8 secretion. Previous findings showing that the copine family could modulated some key proteins of the NF-KB pathway $[36,45]$. So, we speculated that CPNE7 is the regulator of the NF-KB pathway.

Finally, we investigated the regulatory mechanism involved in the CPNE7 activation of in the NF-KB signalling pathway. p65 is one of the most important transcription factors in the NF-KB pathway that assembles with and binds to the inhibitory IкBa to form a complex, which prevents the complex from translocating into the nucleus [43]. Copine family proteins contain two distinctive domains, a phospholipid-binding domain (C2 domains) of signalling proteins in the $\mathrm{N}$-terminus and a protein interaction domain (von Willebrand factor type A domain, vWA) in the C-terminus $[45,48]$. The underlying mechanism of CPNE7 regulation by the NFKB pathway is poorly reported, but homologues of the copine family (such as copine I and copine III) [45] were reported to degrade IKB [36] and induce end protease processing of the $\mathrm{N}$-terminus of p65 [43]. In our results, p65 was released and translocated into the nucleus in OSCC-MSCs and Iv-CPNE7 and the expression of phosphorylated p65 and IKBa was higher in OSCCMSCs than in OLK-MSCs (in Iv-CPNE7 than in Iv-control). So, it speculated that CPNE7 could promote p65 and IKBa phosphorylation and nuclear translocation of $p 65$. In addition, Nuclelion, CPNE7, and IKBa were interacting with each other by Co-IP tests in our results. Therefore, we inferred that Nuclelion may be the cell surface receptor of CPNE7 CPNE7, Nuclelion-CPNE7-IKBa could promote IKBa phosphorylation and nuclear translocation of p65 and regulated the expression of CXCL8, but the detailed mechanism of downstream targeted genes and binding mode still needs to be explored in the future.

In conclusion, during the malignant transformation of OLK, MSCs induce a pro-metastatic phenotype, which is regulated by CXCL8 in the NF-KB pathway and controlled by the CPNE7-Nuclelion-IKBa complex. Targeted management with CPNE7 modulators in MSCs 
A
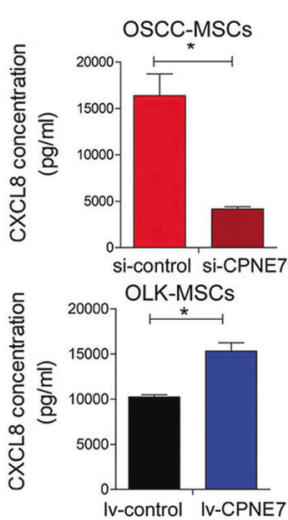

B
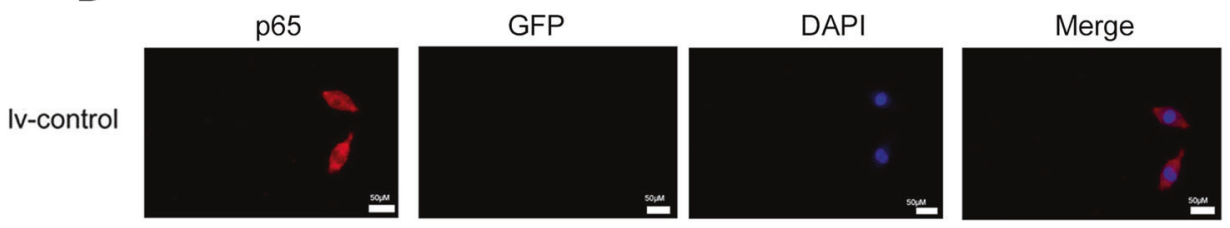

IV-CPNE7
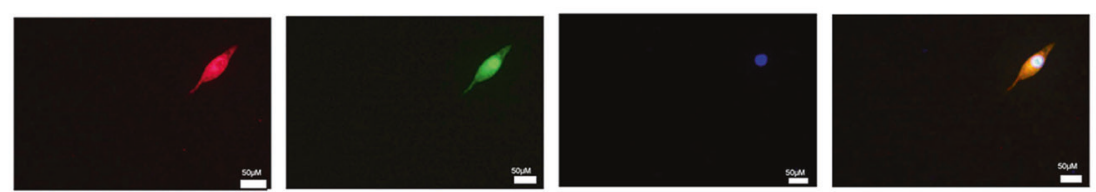

C
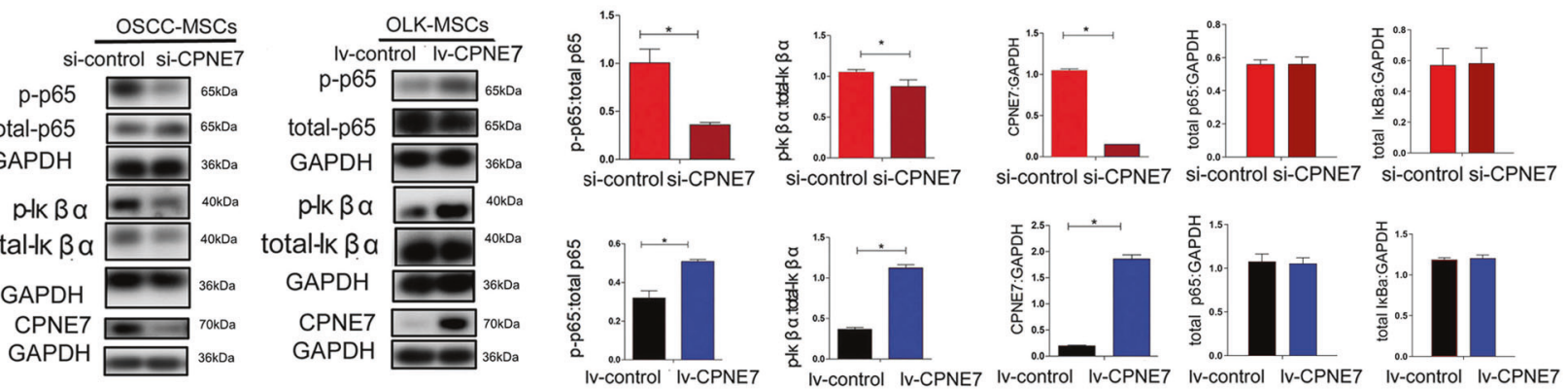

E Input $\frac{\mathrm{IP}}{\text { (GFP) }}$

IB: CPNE7

IB: total-k $\beta a$

IB: GFP
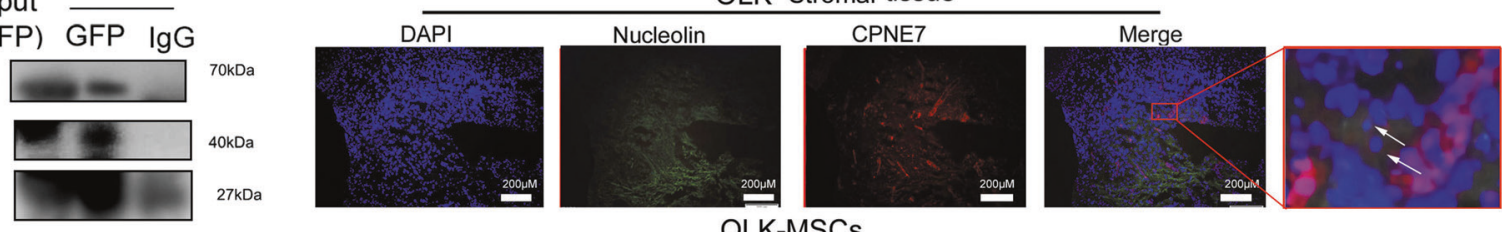

Input IP

total-IK $\beta \alpha$ total-k $\beta a \lg G$

IB: Nucleolin

IB: CPNE7

IB: total--к $\beta a$

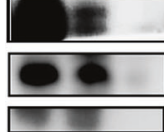

$100 \mathrm{kDa}$
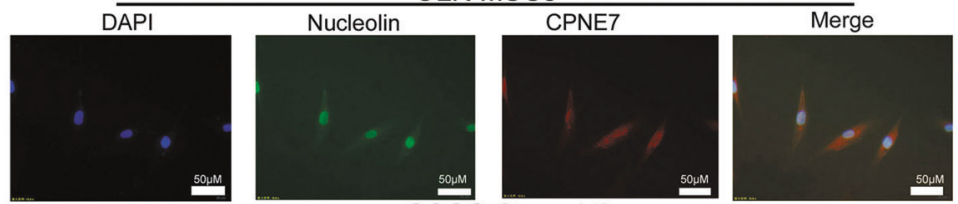

$40 \mathrm{kDa}$

IB: GFP

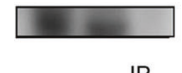

$27 \mathrm{kDa}$

F

Input IP

(Nucleolin) IgG Nucleolin

IB: Nucleolin

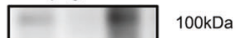

IB: CPNE7

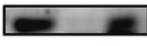

$70 \mathrm{kDa}$
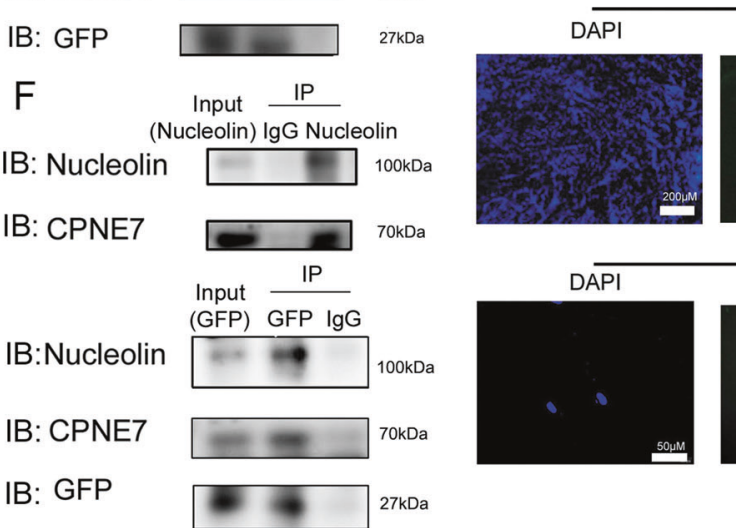

Nucleolin

SCC-Stromal tissue
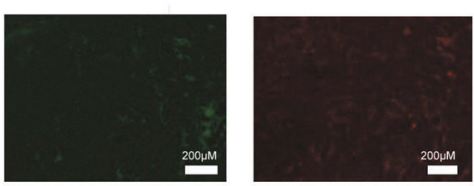

Merge
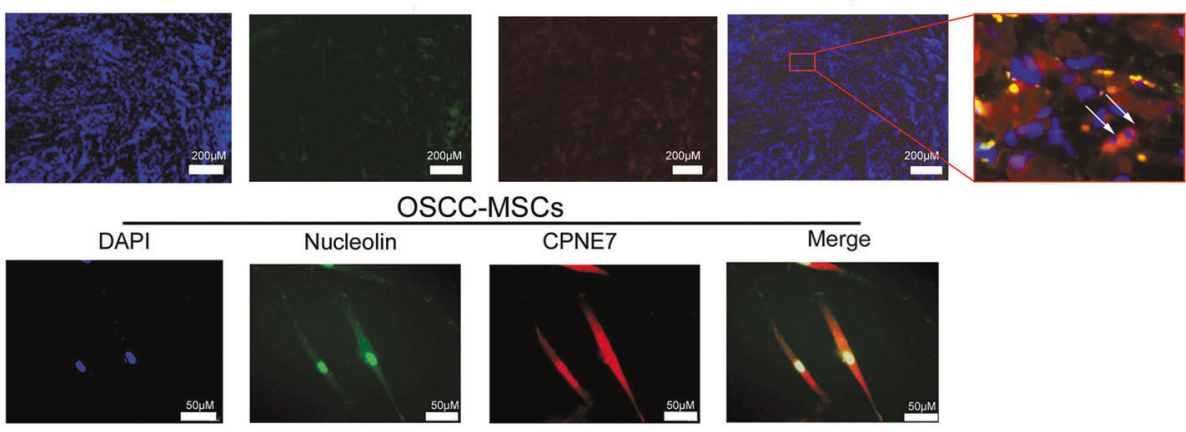

Fig. 6 CPNE7 could enhance the secretion of CXCL8 and interact with IKßa through the NF-kB pathway. A Overexpression and knockdown of CPNE7 in MSCs regulated CXCL8 secretion. B Immunofluorescence on overexpression of CPNE7 (GFP-tagged CPNE7) in OSCC-MSCs was performed to detect the localization of p65 (red) and GFP (green) were stained with DAPI (blue). C Western blot was used to measure the difference in the expression of p65 and $1 \kappa B \alpha$ (NF- $\mathrm{kB}$ pathway) in CPNE7-overexpressing OLK-MSCs and CPNE7-knockdown OSCC-MSCs. D Immunoprecipitated (IP) CPNE7 and whole-cell lysates (Input) were analysed by immunoblotting (IB) with anti- IKB $\alpha$, Nucleolin, CPNE7, and GFP antibodies. E Co-localization of CPNE7 and Nucleolin in tissue and MSCs. The arrow indicated the co-expression of CPNE and Nucleolin in the same cells of the tissue. F Immunoprecipitated (IP) CPNE7 and whole-cell lysates (Input) were analysed by immunoblotting (IB) with antiCPNE7, Nucleolin, and GFP antibodies. The tests were repeated three times. Data were expressed as means \pm SD. ${ }^{*} p<0.05$. 


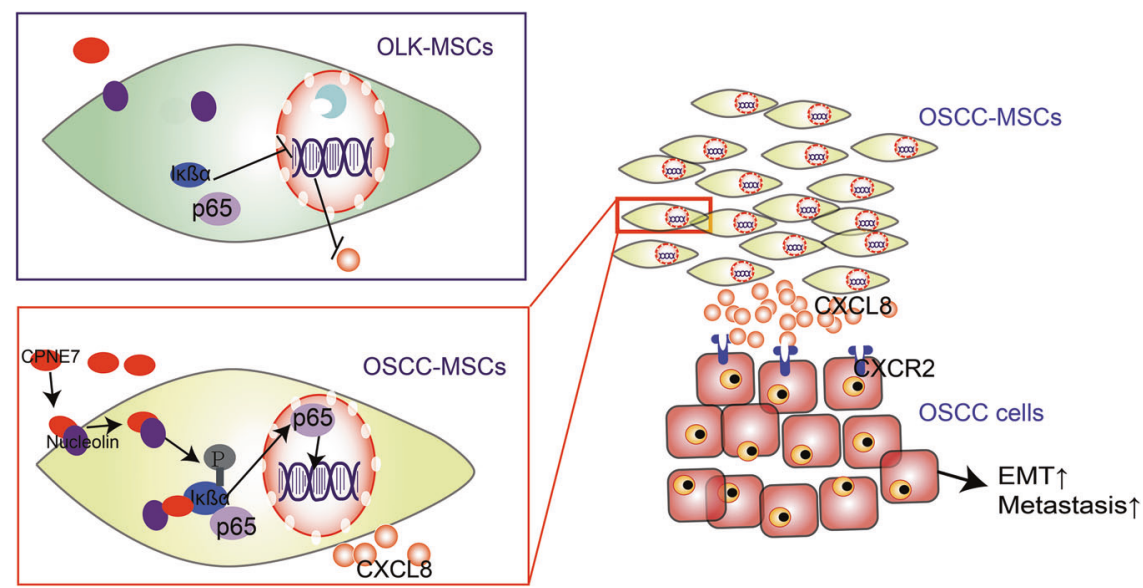

Fig. 7 Schematic depiction. CPNE7 controls CXCL8 secretion through the NF- $\mathrm{kB}$ signalling pathway, promoting the metastasis of OSCC cells in the malignant transformation of OLK to OSCC.

may represent a novel therapeutic approach for the prevention of the malignant transformation of OLK to OSCC.

\section{DATA AVAILABILITY}

Authors can confirm that all relevant data are included in the article and its supplementary information files. The datasets of RNA-sequencing in this study are available in the SRA data (PRJNA665945) (https://www.ncbi.nlm.nih.gov/sra).

\section{REFERENCES}

1. Bray F, Ferlay J, Soerjomataram I, Siegel RL, Torre LA, Jemal A. Global cancer statistics 2018: GLOBOCAN estimates of incidence and mortality worldwide for 36 cancers in 185 countries. CA Cancer J Clin. 2018;68:394-424.

2. Momen-Heravi F, Trachtenberg AJ, Kuo WP, Cheng YS. Genomewide study of salivary microRNAs for detection of oral cancer. J Dent Res. 2014;93:86S-93S.

3. Moradpoor R, Gharebaghian A, Shahi F, Mousavi A, Salari S, Akbari ME, et al. Identification and validation of stage-associated PBMC biomarkers in breast cancer using MS-based proteomics. Front Oncol. 2020;10:1101.

4. Bera A, Lewis SM. Regulation of epithelial-to-mesenchymal transition by alternative translation initiation mechanisms and its implications for cancer metastasis. Int J Mol Sci.2020;21:4075

5. Su PH, Hsu YW, Huang RL, Chen LY, Chao TK, Liao CC, et al. TET1 promotes 5hmCdependent stemness, and inhibits a $5 \mathrm{hmC}$-independent epithelial-mesenchymal transition, in cervical precancerous lesions. Cancer Lett. 2019;450:53-62.

6. Lee DG, Kim HS, Lee YS, Kim S, Cha SY, Ota I, et al. Helicobacter pylori CagA promotes Snail-mediated epithelial-mesenchymal transition by reducing GSK-3 activity. Nat Commun. 2014;5:4423.

7. Dionne KR, Warnakulasuriya S, Zain RB, Cheong SC. Potentially malignant disorders of the oral cavity: current practice and future directions in the clinic and laboratory. Int J. Cancer. 2015;136:503-15.

8. Celentano A, Glurich I, Borgnakke WS, Farah CS. World workshop on oral medicine VII: prognostic biomarkers in oral leukoplakia and proliferative verrucous leukoplakiaA systematic review of retrospective studies. Oral Dis. 2020;27:848-80.

9. Ridge SM, Sullivan FJ, Glynn SA. Mesenchymal stem cells: key players in cancer progression. Mol Cancer. 2017;16:31.

10. Shi Y, Du L, Lin L, Wang Y. Tumour-associated mesenchymal stem/stromal cells: emerging therapeutic targets. Nat Rev Drug Disco. 2017;16:35-52.

11. Ghosh T, Barik S, Bhuniya A, Dhar J, Dasgupta S, Ghosh S, et al. Tumor-associated mesenchymal stem cells inhibit naïve $T$ cell expansion by blocking cysteine export from dendritic cells. Int J Cancer. 2016;139:2068-81.

12. Li W, Han Y, Zhao Z, Ji X, Wang X, Jin J, et al. Oral mucosal mesenchymal stem cellderived exosomes: a potential therapeutic target in oral premalignant lesions. Int J Oncol. 2019;54:1567-78.

13. Laplagne C, Domagala M, Le Naour A, Quemerais C, Hamel D, Fournié J-J, et al. Latest advances in targeting the tumor microenvironment for tumor suppression. Int J Mol Sci. 2019;20:4719.

14. Quail DF, Joyce JA. Microenvironmental regulation of tumor progression and metastasis. Nat Med. 2013;19:1423-37.

15. Wörner PM, Schächtele DJ, Barabadi Z, Srivastav S, Chandrasekar B, Izadpanah R, et al. Breast tumor microenvironment can transform naive mesenchymal stem cells into tumor-forming cells in nude mice. Stem Cells Dev. 2019;28:341-52.
16. Yang $Y$, Otte $A$, Hass R. Human mesenchymal stroma/stem cells exchange membrane proteins and alter functionality during interaction with different tumor cell lines. Stem Cells Dev. 2015;24:1205-22.

17. Berger $L$, Shamai $Y$, Skorecki KL, Tzukerman M. Tumor specific recruitment and reprogramming of mesenchymal stem cells in tumorigenesis. Stem Cells. 2016;34:1011-26.

18. Coffman LG, Pearson AT, Frisbie LG, Freeman Z, Christie E, Bowtell DD, et al. Ovarian carcinoma-associated mesenchymal stem cells arise from tissue-specific normal stroma. Stem Cells. 2019;37:257-69.

19. Orciani M, Caffarini M, Lazzarini R, Delli Carpini G, Tsiroglou D, Di Primio R, et al. Mesenchymal stem cells from cervix and age: new insights into $\mathrm{CIN}$ regression rate. Oxid Med Cell Longev. 2018;2018:1-12.

20. Plava J, Cihova M, Burikova M, Bohac M, Adamkov M, Drahosova S. et al. Permanent pro-tumorigenic shift in adipose tissue-derived mesenchymal stromal cells induced by breast malignancy. Cells. 2020;9:480

21. Muller S. Oral epithelial dysplasia, atypical verrucous lesions and oral potentially malignant disorders: focus on histopathology. Oral Surg Oral Med Oral Pathol. Oral. Radio. 2018;125:591-602.

22. Thompson L. World Health Organization classification of tumours: pathology and genetics of head and neck tumours. Ear Nose Throat J. 2006;85:74.

23. Dominici $M$, Le Blanc $K$, Mueller I, Slaper-Cortenbach I, Marini F, Krause D, et al. Minimal criteria for defining multipotent mesenchymal stromal cells. the international society for cellular therapy position statement. Cytotherapy 2006;8:315-7.

24. Ji X, Zhang Z, Han Y, Song J, Xu X, Jin J, et al. Mesenchymal stem cells derived from normal gingival tissue inhibit the proliferation of oral cancer cells in vitro and in vivo. Int J. Oncol. 2016;49:2011-22.

25. Li YY, Zhou CX, Gao Y. Podoplanin promotes the invasion of oral squamous cell carcinoma in coordination with MT1-MMP and Rho GTPases. Am. J. Cancer Res. 2015;5:514-29.

26. Varghese F, Bukhari AB, Malhotra R, De A. IHC Profiler: an open source plugin for the quantitative evaluation and automated scoring of immunohistochemistry images of human tissue samples. PLoS One. 2014;9:e96801.

27. Rotondi M, Coperchini F, Pignatti P, Sideri R, Groppelli G, Leporati P, et al. Interferon-gamma and tumor necrosis factor-alpha sustain secretion of specific CXC chemokines in human thyrocytes: a first step toward a differentiation between autoimmune and tumor-related inflammation? J Clin Endocrinol Metab. 2013;98:308-13. https://doi.org/10.1210/jc.2012-2555

28. Cheng J, Li Y, Liu S, Jiang Y, Ma J, Wan L, et al. CXCL8 derived from mesenchymal stromal cells supports survival and proliferation of acute myeloid leukemia cells through the PI3K/AKT pathway. FASEB J. 2019;33:4755-64.

29. Paczek S, Lukaszewicz-Zajac M, Gryko M, Mroczko P, Kulczynska-Przybik A, Mroczko B. CXCL-8 in preoperative colorectal cancer patients: significance for diagnosis and cancer progression. Int J Mol Sci. 2020;21:2040.

30. Yang JW, Murray B, Barbier-Torres L, Liu T, Liu Z, Yang H, et al. The mitochondrial chaperone Prohibitin 1 negatively regulates interleukin-8 in human liver cancers. J Biol Chem. 2019;294:1984-96.

31. Liubomirski Y, Lerrer S, Meshel T, Morein D, Rubinstein-Achiasaf L, Sprinzak D, et al. Notch-mediated tumor-stroma-inflammation networks promote invasive properties and CXCL8 expression in triple-negative breast cancer. Front Immunol. 2019;10:804.

32. Katanov C, Lerrer S, Liubomirski Y, Leider-Trejo L, Meshel T, Bar J, et al. Regulation of the inflammatory profile of stromal cells in human breast cancer: prominent roles for TNF-alpha and the NF-kappaB pathway. Stem Cell Res Ther. 2015;6:87. 
33. Li X, Wang S, Zhu R, Li H, Han Q, Zhao RC. Lung tumor exosomes induce a proinflammatory phenotype in mesenchymal stem cells via NFkappaB-TLR signaling pathway. J Hematol Oncol. 2016;9:42.

34. Kang DW, Park MK, Oh HJ, Lee DG, Park SH, Choi KY, et al. Phospholipase D1 has a pivotal role in interleukin-1 -driven chronic autoimmune arthritis through regulation of NF- B, Hypoxia-Inducible Factor 1, and FoxO3a. Mol Cell Biol. 2013;33:2760-72.

35. Ji X, Li L, Lu P, Li X, Tian D, Liu M. NLRP6 exerts a protective role via NF-kB with involvement of CCL20 in a mouse model of alcoholic hepatitis. Biochem Biophys Res Commun. 2020;528:485-92.

36. Tomsig JL, Sohma H, Creutz CE. Calcium-dependent regulation of tumour necrosis factor-alpha receptor signalling by copine. Biochem J. 2004;378:1089-94.

37. Chen R, Cheng $Q$, Owusu-Ansah KG, Song G, Jiang D, Zhou $L$, et al. NKILA, a prognostic indicator, inhibits tumor metastasis by suppressing NF-kappaB/Slug mediated epithelial-mesenchymal transition in hepatocellular carcinoma. Int J Biol Sci. 2020;16:495-503. https://doi.org/10.7150/ijbs.39582

38. Scheidereit C. IkappaB kinase complexes: gateways to NF-kappaB activation and transcription. Oncogene 2006;25:6685-705.

39. Seo YM, Park SJ, Lee HK, Park JC. Copine-7 binds to the cell surface receptor nucleolin, and regulates ciliogenesis and Dspp expression during odontoblast differentiation. Sci Rep. 2017;7:11283.

40. Li W, Zhang X, Wu F, Zhou Y, Bao Z, Li H, et al. Gastric cancer-derived mesenchymal stromal cells trigger M2 macrophage polarization that promotes metastasis and EMT in gastric cancer. Cell Death Dis. 2019;10:918.

41. Chang MC, Chiang CP, Lin CL, Lee JJ, Hahn LJ, Jeng JH. Cell-mediated immunity and head and neck cancer: with special emphasis on betel quid chewing habit. Oral Oncol. 2005;41:757-75.

42. Punyani SR, Sathawane RS. Salivary level of interleukin-8 in oral precancer and oral squamous cell carcinoma. Clin Oral Investig. 2013;17:517-24.

43. Ramsey CS, Yeung F, Stoddard PB, Li D, Creutz CE, Mayo MW. Copine-I represses NF-kappaB transcription by endoproteolysis of p65. Oncogene 2008;27:3516-26.

44. Oh HJ, Choung HW, Lee HK, Park SJ, Lee JH, Lee DS, et al. CPNE7, a preameloblast-derived factor, regulates odontoblastic differentiation of mesenchymal stem cells. Biomaterials. 2015;37:208-17.

45. Savino $M$, d'Apolito $M$, Centra $M$, van Beerendonk HM, Cleton-Jansen AM Whitmore SA, et al. Characterization of copine VII, a new member of the copine family, and its exclusion as a candidate in sporadic breast cancers with loss of heterozygosity at 16q24.3. Genomics. 1999;61:219-26.

46. Bensidhoum M, Chapel A, Francois S, Demarquay C, Mazurier C, Fouillard L, et al. Homing of in vitro expanded Stro-1- or Stro-1+ human mesenchymal stem cells into the NOD/SCID mouse and their role in supporting human CD34 cell engraftment. Blood. 2004;103:3313-9.

47. Seo B-M, Miura M, Gronthos S, Mark Bartold P, Batouli S, Brahim J, et al. Investigation of multipotent postnatal stem cells from human periodontal ligament. Lancet. 2004;364:149-55.

48. Perestenko $P$, Watanabe $M$, Beusnard-Bee $T$, Guna $P$, Mcllhinney J. The second C2-domain of copine-2, copine- 6 and copine-7 is responsible for their calciumdependent membrane association. FEBS J. 2015;282:3722-36.

\section{ACKNOWLEDGEMENTS}

This work was supported by Shandong Natural Science Foundation (No. ZR2019PH004), grants from the School and Hospital of Stomatology of Shandong University Youth Fund (No.2018QNJJ04), and the start-up fund for the introduction of talents to Jinan Central Hospital (No. YJRC2021002). We acknowledge Prof. Yixiang Wang (Peking University School and Hospital of Stomatology) for his wonderful suggestions.

\section{AUTHOR CONTRIBUTIONS}

$\mathrm{QF}, \mathrm{XJ}$, and $\mathrm{HL}$ conceived and designed the project. $\mathrm{XJ}$ performed most of the experiments and analyzed the data. TS designed and analyzed the RNA-seq data. HQ SX, and LS provided technical assistance. SX and LS collected the samples. LW performed the RNA-seq experiment. XJ, QF wrote and revised the paper.

\section{COMPETING INTERESTS}

The authors declare no competing interests.

\section{ADDITIONAL INFORMATION}

Supplementary information The online version contains supplementary material available at https://doi.org/10.1038/s41420-021-00684-w.

Correspondence and requests for materials should be addressed to Xiaoli Ji, Hongwei Liu or Qiang Feng.

Reprints and permission information is available at http://www.nature.com/ reprints

Publisher's note Springer Nature remains neutral with regard to jurisdictional claims in published maps and institutional affiliations.

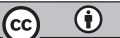

Open Access This article is licensed under a Creative Commons Attribution 4.0 International License, which permits use, sharing, adaptation, distribution and reproduction in any medium or format, as long as you give appropriate credit to the original author(s) and the source, provide a link to the Creative Commons license, and indicate if changes were made. The images or other third party material in this article are included in the article's Creative Commons license, unless indicated otherwise in a credit line to the material. If material is not included in the article's Creative Commons license and your intended use is not permitted by statutory regulation or exceeds the permitted use, you will need to obtain permission directly from the copyright holder. To view a copy of this license, visit http://creativecommons. org/licenses/by/4.0/.

(c) The Author(s) 2021 